\title{
PLASMA COPEPTIN IN PRETERM INFANTS - A HIGHLY SENSITIVE MARKER OF FETAL
} AND NEONATAL STRESS

\author{
S. Wellmann ${ }^{1}$, J. Benzing ${ }^{2}$, F. Achini ${ }^{2}$, G. Szinnai ${ }^{3}$, C. Bührer ${ }^{4}$, H.U. Bucher ${ }^{1}$ \\ ${ }^{1}$ Neonatology, University Hospital Zürich, Zürich, ${ }^{2}$ Neonatology, ${ }^{3}$ Endocrinology, University Children's \\ Hospital Basel, UKBB, Basel, Switzerland, ${ }^{4}$ Neonatology, Charité University Medical Center, Berlin, \\ Germany
}

Background: Copeptin is a stable by-product of arginine-vasopressin (AVP) synthesis and reflects its secretion by the pituitary. We investigated perinatal factors affecting copeptin concentrations in preterm infants at birth and at 3 days of life.

Methods: A prospective cross-sectional study at two Swiss university hospitals was performed. 167 preterm infants were enrolled, 59 infants born between 24 and 31 weeks gestational age, 50 infants between 32 and 34 weeks, and 58 between 35 and 36 weeks. Plasma copeptin concentrations were determined by a CTproAVP-Luminescence-immunoassay in umbilical cord blood at birth and in blood samples obtained at 2-3 days of life.

Results: Copeptin at birth was significantly higher in preterm infants born vaginally (median [range] 366 [12900] $\mathrm{pmol} / \mathrm{L}, \mathrm{n}=43)$ than those born by caesarean section $(6.9$ [2-1580], $\mathrm{n}=124)$. In infants born after caesarean without prior labour $(n=66)$, small for gestational age $(<5$ th percentile $)$, suspect fetal heart rate, compromised placental perfusion, and chorioamnionitis were each associated with significantly elevated cord copeptin. Copeptin at 3 days of life was not associated with cord blood copeptin but inversely related to gestational age (Rs -0.6, $\mathrm{p}<0.001)$ and birth weight (Rs $-0.612, \mathrm{p}<0.001)$. Day 3 copeptin increased alongside the level of mechanical respiratory support.

Conclusion: Copeptin is a highly sensitive marker of fetal and neonatal stress. 